\title{
Nontuberculous Mycobacterial Pulmonary Disease in an Immunocompromised Patient
}

\author{
Ranjan K. Singh ${ }^{1}$, Dipak Kumar ${ }^{1}$ \\ 1. Internal Medicine, Anti-Retroviral Therapy Centre, District Hospital, Khagaria, IND
}

Corresponding author: Ranjan K. Singh, dr_ranjankumarsingh@yahoo.com

\begin{abstract}
In this article, we discuss the nontuberculous mycobacterial pulmonary disease in a 40-year-old HIVseropositive female patient. The patient has a history of pulmonary tuberculosis, experienced two years ago. At the time, she was treated successfully with anti-tuberculous therapy. A chest x-ray (CXR) and computed tomography (CT) scan of the chest showed a thin-walled cavitary lesion in the right lung. In addition, the tree-in-bud sign, indicative of airway obstruction, was present on CT imaging. Fluorescence microscopy using auramine staining showed acid-fast bacilli (AFB) in sputum smears on more than two samples. Mycobacterium tuberculosis was not detected in the nucleic acid amplification test in the same sample. The AFB identified were mycobacteria other than tubercle bacilli, i.e., nontuberculous mycobacteria, that cause cavitary lung disease. Culture in liquid media and subsequent molecular analysis showed Mycobacterium avium complex (MAC). The patient is now being treated with a multidrug regimen of antibiotics and has improved, with documented sputum conversion.
\end{abstract}

Categories: HIV/AIDS, Infectious Disease, Pulmonology

Keywords: hiv, ntm, mai

\section{Introduction}

Nontuberculous mycobacteria (NTM) are a group of bacilli, other than tuberculous, containing more than 160 species. Of these species, Mycobacterium avium complex (MAC), M. kansasii, M. abscessus, and M. xenopi frequently cause pulmonary disease in patients of compromised immune status.

Received 09/15/2019

Review began 09/18/2019 Review ended 10/29/2019 Published 11/03/2019

\section{() Copyright 2019}

Singh et al. This is an open access article distributed under the terms of the Creative Commons Attribution License CC-BY 3.0., which permits unrestricted use, distribution, and reproduction in any medium, provided the original author and source are credited.
NTM are ubiquitous in the soil and water. In contrast to M. tuberculosis, NTM is not transmitted through person-to-person contact but, rather, spread from soil and water through aerosolized droplets. The prevalence of nontuberculous mycobacteria pulmonary disease (NTM-PD) varies from one region to another; for example, there are 5.9 cases per 100,000 population in Spain, 6.5 cases per 100,000 population in the United Kingdom (UK), and 1.3 cases per 100,000 population in France [1]. The disease happens to be a frequently missed diagnosis because the clinical features of NTM-PD are indistinguishable from that of pulmonary tuberculosis. Also, sputum smear microscopy does not distinguish NTM from M. tuberculosis, as bacilli in both cases are acid-fast and morphologically similar in appearance. Adhikaram found that patients with NTM-PD have been erroneously diagnosed as having tuberculosis and multidrug-resistant tuberculosis in 4.5 to $15 \%$ and 18 to $20 \%$ cases, respectively [2]. Guidelines for detecting NTM-PD were established in 2007 by the American Thoracic Society and the Infectious Diseases Society of America [3]. The guidelines include details of clinical presentation, such as fever, cough, hemoptysis, and breathlessness, along with radiological features, such as cavitary lesions, bronchiectatic changes, and nodules. Pleural effusion and hilar lymphadenopathy are uncommon in NTM. Computed tomography (CT) of the chest shows a thinwalled cavity, multiple nodules, and the tree-in-bud sign. Sputum analysis from samples obtained on two occasions or samples from bronchoalveolar lavage on one occasion should show NTM.

\section{Case Presentation}

A 40-year-old human immunodeficiency virus (HIV)-seropositive female was complaining of fever, cough, and hemoptysis for two months. She had been taking antiretroviral therapy (tenofovir, lamivudine, and efavirenz) for the past seven years. Her recent CD4 cell count and viral load were 476/ $\mathrm{mm}^{3}$ and 1442 copies/ml respectively. She had pulmonary tuberculosis (drug-sensitive Mycobacterium tuberculosis) two years ago and responded completely to nine months of anti-tuberculous treatment. A recent clinical examination revealed diffuse rhonchi on the right side of her chest. Chest x-ray (CXR) showed a cavitary lesion in the right lung field with homogenous opacity in the middle zone of her right lung (Figure 1). 


\section{Cureus}

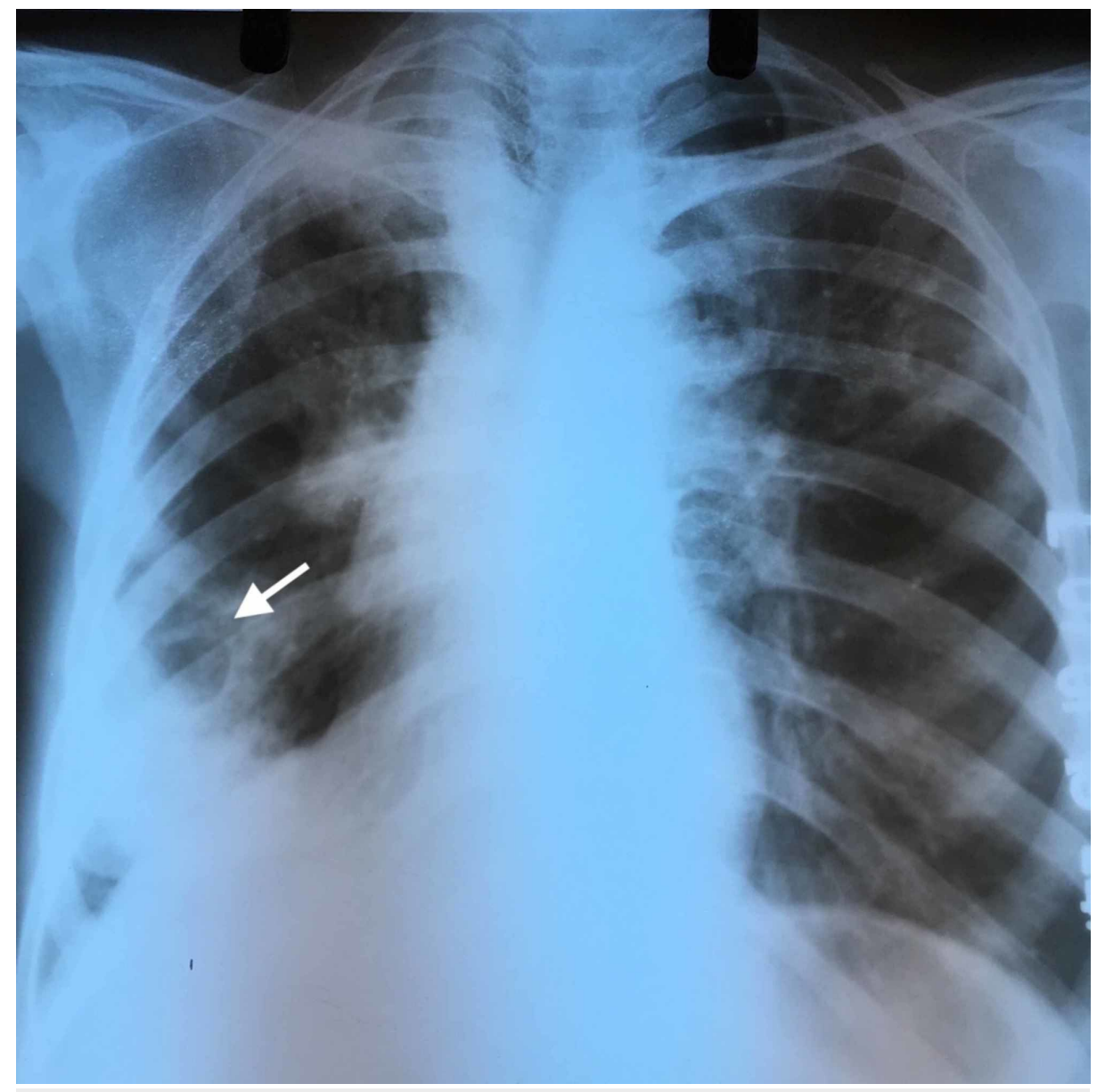

FIGURE 1: Chest X-ray showing a cavitary lesion in the lower zone of the right lung field

A CT scan of her chest showed a cavitary lesion in the right middle lung and a tree-in-bud appearance in the left lung (lingual) (Figure 2).

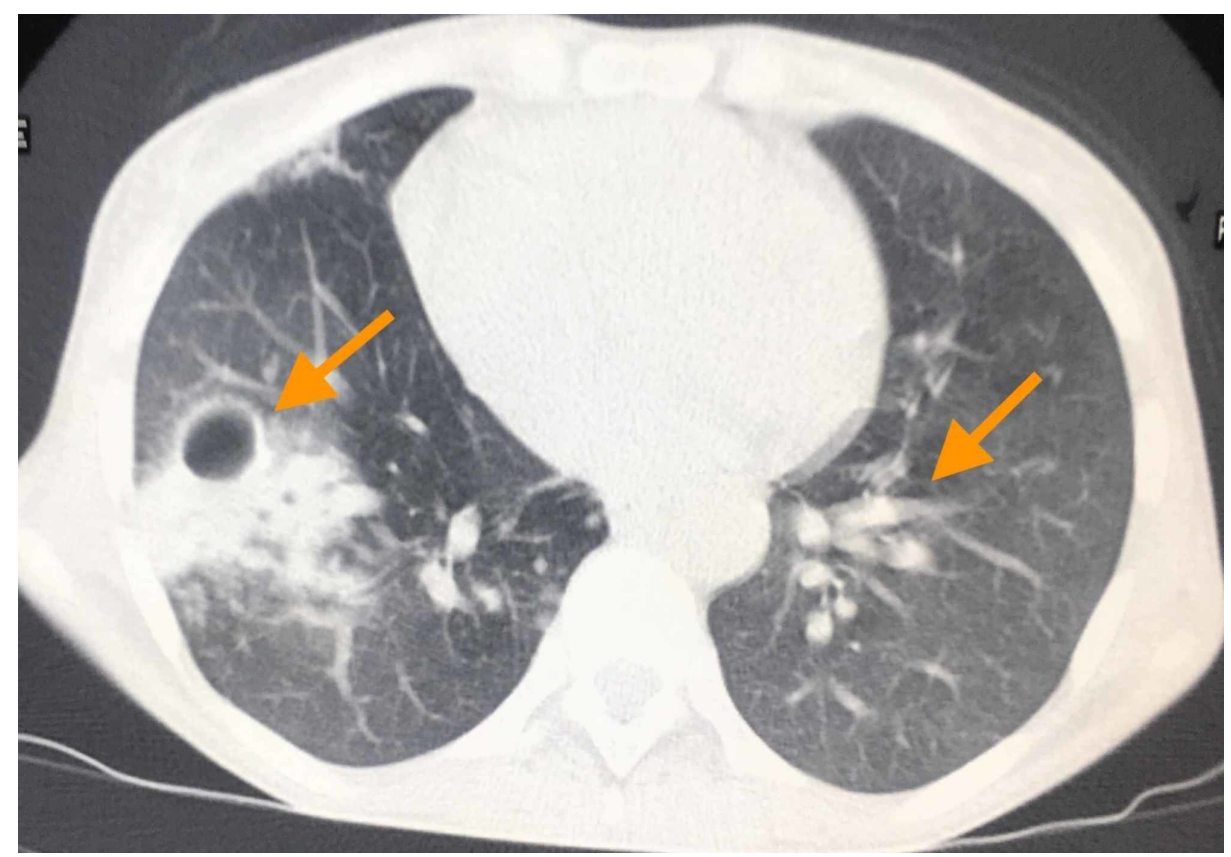

FIGURE 2: CT scan showing cavitary lesion in the right lung and tree-in- 


\section{Cureus}

\section{bud sign in the left lung}

Sputum collected in the morning was subjected to fluorescence microscopy after auramine staining and nucleic acid amplification test (NAAT) for M. tuberculosis. Microscopy showed acid-fast bacilli (AFB) (Figure 3) but the NAAT was negative for M. tuberculosis.

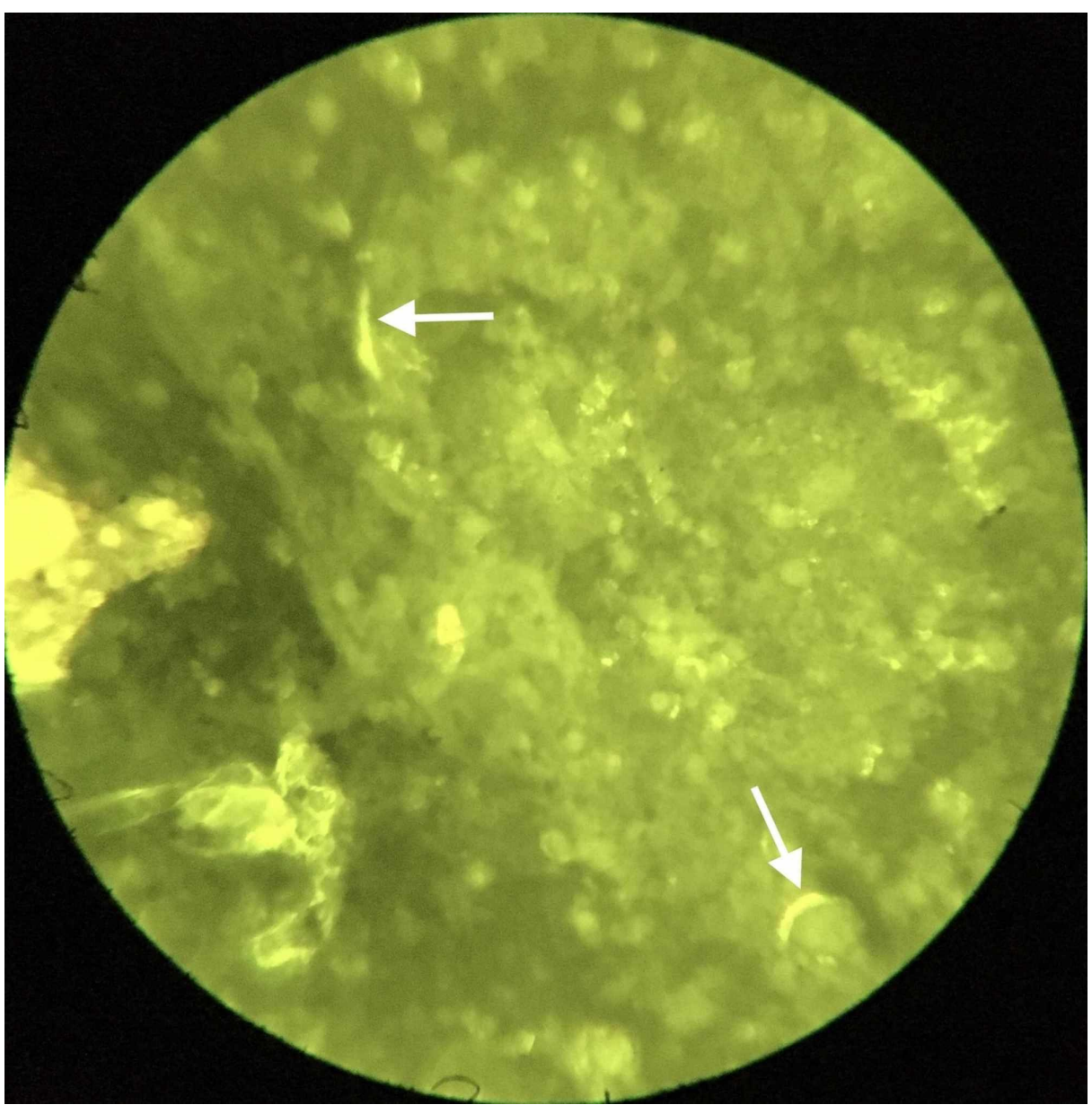

\section{FIGURE 3: Sputum smear with auramine staining under fluorescence} microscopy (magnification 200X) showing acid-fast bacilli

Again, sputum was collected on the second day and examined, with similar findings as for the previous sputum collection. Culture on liquid media showed NTM, which were sent to the National Reference Laboratory for mycobacterial diseases in Delhi, for species identification and determination of sensitivity to drugs. The species was identified as MAC.

The patient was treated with azithromycin $500 \mathrm{mg}$ once daily (OD), rifampicin 450mg OD, ethambutol 600mg OD, and amikacin (Mikacin) 750mg intravenously (IV) three times a week for two months, at which point in time the Mikacin injection was stopped. Sputum conversion was detected after four months and treatment continued for a further 12 months after sputum conversion.

\section{Discussion}

Like solid organ transplant recipient patients, HIV-seropositive patients have an increased risk for infection with NTM because of depressed cell-mediated immunity. Several preexisting lung diseases such as cystic fibrosis, bronchiectasis, prior pulmonary tuberculosis, chronic obstructive pulmonary disease, and pneumoconiosis predispose to NTM. HIV infection and prior pulmonary tuberculosis led to the acquisition of NTM-PD in this case.

Possible differential diagnoses for a thin-walled cavitary pulmonary lesion in HIV-seropositive cases include tuberculosis, invasive aspergillosis, Pneumocystis jirovecii pneumonia, endemic fungal infections, rheumatoid arthritis, as well as nontuberculous mycobacterium. CT images show a cavitary lesion in $46 \%$, 
nodules in $29 \%$, and the tree-in-bud sign in $21 \%$ of cases of NTM-PD [4]. In our case, a thin built middle age lady has tree-in-bud in lingula and cavitary lesion in the right middle lobe on CT imaging. A similar pattern of tree-in-bud sign localized in right middle lobe or lingula in an immunocompetent middle age lady has been described as "lady Windermere syndrome" 25 years back. The tree-in-bud sign is not pathognomonic to NTM-pulmonary disease because this sign can be found in infective bronchiolitis, cystic fibrosis, endobronchial tuberculosis, allergic bronchopulmonary aspergillosis, and rheumatoid arthritis [5].

Microscopic detection of AFB in sputum smear and a simultaneous negative NAAT for M. tuberculosis strongly indicate NTM-PD [6]. However, a culture of the sputum specimen is still regarded as the gold standard method for microbiological confirmation.

Dissemination of NTM infection depends on CD4 T cell status i.e. $<50 / \mathrm{mm}^{3}$ in HIV patients. Prompt initiation of antiretroviral drugs with the maintenance of CD4 T cells $>100 / \mathrm{mm}^{3}$ prevents dissemination. NTM infections were observed among patients who have viral load $>1000$ copies/ml, however, there is statistically no difference in the incidence of NTM infections among different baseline viral loads [7].

\section{Conclusions}

The prevalence of NTM-PD varies geographically, and the disease is easily missed, commonly diagnosed instead as pulmonary tuberculosis and drug-resistant pulmonary tuberculosis. Fluorescence microscopy of sputum smear and simultaneous NAAT (of the same sample) provide innovation in the diagnosis of NTM$\mathrm{PD}$. A culture of sputum for species identification and determining drug sensitivity is regarded as the gold standard method for confirmation of the diagnosis of NTM. NTM dissemination depends on the lower CD4 T cell status of the patient.

\section{Additional Information}

\section{Disclosures}

Human subjects: Consent was obtained by all participants in this study. N/A issued approval N/A. Patient consent was obtained prior to submitting it for publication. Conflicts of interest: In compliance with the ICMJE uniform disclosure form, all authors declare the following: Payment/services info: All authors have declared that no financial support was received from any organization for the submitted work. Financial relationships: All authors have declared that they have no financial relationships at present or within the previous three years with any organizations that might have an interest in the submitted work. Other relationships: All authors have declared that there are no other relationships or activities that could appear to have influenced the submitted work.

\section{References}

1. Wagner D, van Ingen J, Adjemian J, et al.: Annual prevalence and treatment estimates of nontuberculous mycobacteria-pulmonary disease in Europe: a NTM-NET collaborative study. Eur Respir J. 2014, 44:P1067.

2. Adikaram CP: Overview of nontuberculosis mycobacterial lung diseases . Mycobacterium - Research and Development. IntechOpen, 2018. 257-286. 10.5772/intechopen.73542

3. Griffith DE, Aksamit T, Brown-Elliott BA, et al.: An official ATS/IDSA statement: diagnosis, treatment and prevention of nontuberculous mycobacterial diseases. Am J of Respir and Crit Care Med. 2007, 175:367-416. 10.1164/rccm.200604-571ST

4. Schiff HF, Jones S, Achaiah A, Pereira A, Stait G, Green B: Clinical relevance of non-tuberculous mycobacteria isolated from respiratory specimens: seven year experience in a UK hospital. Sci Rep. 2019, 9:1730. https://www.nature.com/articles/s41598-018-37350-8

5. Gosset N, Bankier AA, Eisenberg RL : Tree-in-bud pattern. AJR Am J Roentgenol. 2009, 193:472-477. 10.2214/AJR.09.3401

6. Wassilew N, Hoffmann H, Andejak C, Lange C: Pulmonary disease caused by non-tuberculous mycobacteria . Respiration. 2016, 91:386-409. 10.1159/000445906

7. Yangco BG, Buchacz K, Baker R, Palella FJ, Armon C, Brooks JT : Is primary mycobacterium avium complex prophylaxis necessary in patients with $\mathrm{CD} 4<50$ cells $/ \mu \mathrm{L}$ who are virologically suppressed on cART?. AIDS Patient Care STDS. 2014, 28:280-283. 10.1089/apc. 2013.0270 